\title{
The diverse metabolic heterogeneity of stem cells in a BRCA+/- breast cancer population
}

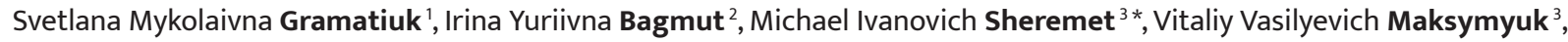
Volodimir Volodimirovich Tarabanchuk ${ }^{3}$, Petro Vasilyevich Moroz $^{3}$

\section{Author Affiliations:}

1. Institute of Cellular Biorehabilitation, Ukraine Association of Biobank Kharkiv, Ukraine

2. Kharkiv Medical Academy of Postgraduate Education, Kharkiv, Ukraine

3. Surgical Department No. 1, Bukovinian State Medical University, Chernivtsi, Ukraine

\author{
* Corresponding Author: \\ Michael Ivanivich Sheremet, \\ Surgery Department No.1 \\ of Bukovinian State Medical \\ University, Holovnastr., 191, \\ 58018, Chernivtsi, Ukraine. \\ Phone: 0956064607. \\ E-mail: mihayl71@gmail.com
}

DOI

10.25122/jml-2020-0105

\section{Dates}

Received: 24 March 2021

Accepted: 6 May 2021 cells and their microenvironment, which behave like a complex and heterogeneous metabolic ecosystem, where cancer cells can reprogram their metabolism as a result of interaction with the components of the microenvironment. The study was based on cancer stem cells (CSG) that were isolated from breast tumors by magnetic separation (AutoMACS). We used spectrophotometric methods for the measurement of aldehyde dehydrogenase (ALDH) enzymatic activity. For these experiments, we used breast cancer and normal stem cell lines. Analyses showed that the proportion of BRCA+ CSC cells was in accordance with the relatively low percentages of CSCs in BRCA+ tumors. ALHD was significantly higher in the CSCs-high BRCA+ breast cancer and CSCs-low BRCA- breast cancer cells, compared with the CSCis-low BRCA+ breast cancer. Breast cancer from BRCA mutation carriers harbor more "high-energy" cell sub-populations than "low-energy" and have their more aggressive phenotype. Key oncogenic pathways known to be dysregulated in breast cancer also regulate stem-cell behavior.

KEYWORDS: stem-cell behavior, mitochondria, BRCAl gene, MCF-7 breast cancer cells, breast cancers.

\section{INTRODUCTION}

In each cellular process stage, from signal transduction of cellular metabolism to cell death, mitochondria play a key role. Taking into account that these features are essential for cancer growth, the application point for anti-cancer therapy can be focused on the mitochondria. It should also be mentioned that mitochondria participate not only in the regulation of stem cell identity and differentiation but also in outcomes. The critical role of mitochondria has also been shown in malignant stem-like cells termed cancer stem cells (CSCs), which are involved in the progression and resistance of different kinds of tumors [1-4].

Breast cancers belong to diverse tissues represented by epithelial cancer cells and an abnormal tumor microenvironment including blood and lymphatic tumor vessels, an extracellular matrix (ECM), and non-cancer stromal cells constituted by endothelial and immune cells, pericytes and mesenchymal stem cells (MSCs), activated adipocytes and cancer-associated fibroblasts (CAFs).

However, cancer cells represent a tissue with a similar complex and heterogeneous metabolic ecosystem with the ability to reprogram their metabolism as a result of interaction with microenvironment factors [5-8]. Generally, breast cancer cells can be divided into three groups: "bulk" cancer cells, which represent $85-95 \%$ of the population, progenitor cells (5\%), and cancer stem cells (CSCs) (1\%). The 


\section{JOURNAL of MEDICINE and LIFE}

most dangerous of them are progenitor cells and CSCs because they can act as tumor-initiating cells (TICs) in vivo and are responsible for metastasis. On the contrary, "bulk" cancer cells belong to a cell population with low tumorigenic potential.

Heterozygous germline mutations in the BRCAl gene predispose women to up to an $80 \%$ lifetime risk of developing breast cancer [6, 9-14]. Most of these tumors have the basal phenotype, characterized by the expression of myoepithelial markers, but lack the expression of ER, PR, and ERBB2 receptors.

Nevertheless, one of the fantastic properties of breast cancer cells continues to be their metabolic plasticity. Notably, the phenomenon known as the Warburg effect relates to a shift from mitochondrial oxidative phosphorylation (OXPHOS) to glycolysis in the presence of oxygen in breast cancer cells which is the most critical process for energy-saving and rapid proliferation [15-19].

\section{Clinical implications of the cancer stem-cell hypothesis}

The cancer stem-cell hypothesis has fundamental implications for breast carcinogenesis as well as important clinical implications for prevention and therapy. In our research, we wanted to test the following hypothesis:

There are heterogeneous metabolic types in the CSC breast cancer BRCA+/- population;

Clinical, molecular, and pathologic features of breast cancer in BRCAl mutation carriers suggest the possibility that BRCAl may function as a stem-cell regulator.

\section{Study details}

In the first step, stem cells were isolated from the breast cancer BRCA+/- population. In the second step, stem cells from conditionally healthy donors from the Ukrainian Association of Biobank under the CIMBA project were obtained. Lastly, the third step included laboratory in vivo research on the clinical, molecular, and pathologic features CSCs breast cancer in BRCA1 and BRCA2 mutations. Multiple analyses were conducted for CSCs, including the breast cancer BRCA+/- population phenotype, aldehyde dehydrogenase expression, mammosphere formation, and the magnetic-activated cell sorting approach to metabolically fractionate the breast cancer cell population into "low-energy" and "high-energy" cell sub-populations.

\section{MATERIAL AND METHODS}

Isolation of cancer stem cells from primary human BRCA+ and BRCA-breast tumors using magnetic-activated cell sorting (MACS) from patients with breast cancer was performed in the context of a clinical protocol. CSCs were isolated from breast tumors using the autoMACS technology. This was followed by seeding 50,000 mononucleated cells $/ \mathrm{cm}^{2}$ in an RPMI (1x) + GlutaMAX medium (Gibco Life Technologies, Canada) supplemented with 10\% fetal bovine serum (Thermo Fisher Scientific, USA) in a Tissue Culture Dish (BioLite 60mm, Thermo Fisher Scientific, USA) [20].

The cultures were incubated at $37^{\circ} \mathrm{C}, 20 \% \mathrm{O}_{2}, 5 \% \mathrm{CO}_{2}$ using the automatic Fibra Stage system (New Brunswick Scientific, USA). Medium changes were performed twice a week. Two weeks after the initial seeding, primary CSCs colonies were separated after 10 mini-incubations at $37^{\circ} \mathrm{C}$ with Trypsin-EDTA $0.05 \%$ (Gibco Life Technologies, Canada) and replaced with $4000 \mathrm{cells} / \mathrm{cm}^{2}$ in the same medium. Subsequent passages were performed following the same steps. Passages 4-6 were used for all experiments [21].

MACS can be used to select metabolically "low-energy" and "high-energy" cell subpopulations of breast cancer and test this hypothesis [6]. Their energetic state can be analyzed using auto-fluorescence assigned to the endogenous flavin-containing metabolites: flavin mononucleotide (FMN), flavin adenine dinucleotide (FAD) and riboflavin.

We used spectrophotometric methods to measure the effects of siRNA expression, particularly the enzymatic activity of ALDH.

First, from each of the stem cell lines (cancer and normal cells), the lysates were separated according to size in parallel on two $12 \%$ denaturing SDS-polyacrylamide gels (BioRad, Hercules, CA). Afterward, they were electrotransferred onto nitrocellulose membranes and blocked with $5 \%$ milk in tris-buffered saline (TBS). A substrate of cell lysate, $5 \mathrm{mM} \mathrm{NAD+,} 5 \mathrm{mM}$ propionaldehyde and $600 \mu \mathrm{l}$ lysing buffer was incubated using spectrophotometer cuvettes (Dynex Technology, USA) at $37^{\circ} \mathrm{C}$.

In three replicates, the rate of change in absorbance at $340 \mathrm{~nm}$ was assessed over $5 \mathrm{~min}$. The endogenous rate of NAD+ reduction was also monitored in the control reaction without a substrate. The ALDH activity was measured in nmoles/10 cells min [21]. The cells were collected and automatically counted after 72 hours of growth in 6 -well tissue culture plates of triplicates of $2 \times 10$ cells $/ \mathrm{ml} /$ well. The breast cancer and normal stem cell lines were used. Triplicates of 200 cells $/ \mathrm{ml} /$ well for each cell line were cultured in a six-well plate in an RPMI-1640 medium with 10\% Fetal Bovine Serum (FBS). Growth time was 5 days. 
The number of colonies adhered to the bottom of the plate was counted using an inverted microscope. The in vitro scratch wound migration assay was used to count the downregulation of ALDH on cell migration and motility process. From five different cell lines, 23 monolayer confluent cells were received and scratched with the sterile pipette tip. The migration ability of the cells was measured with a digital camera after 20 hours in an RPMI-1640 medium $+10 \%$ FBS. The width of the wound was examined under an inverted microscope.

Scratch wound images were taken straight after the scratch and 20 hours later. The migration distances (in cm) of the cells were measured on printed images taken at the same magnification. The migration distances of the cells and the width of wounds at the beginning and 20 hours later were measured, and the wound healing percentage was evaluated.

\section{Statistical analysis}

Tests were used for the three independent experiments. The results were expressed as mean and standard deviation. Analysis of variance (one-way ANOVA) was performed in combination with Dunnett's post-test for multiple comparisons. Correlations between STAT1 and PLSCR 1 were determined by Pearson's correlation and Spearman's rank correlation test. Differences from the controls were considered significant as *p-0.05, **p-0.001 and***p-0.0001. The GraphPad Prism software (version 5.03) was used for all statistical assessments.

\section{RESULTS AND DISCUSSION}

Isolation of cancer stem cells from primary human BRCA+ and BRCA-breast tumors using MACS were collected in the context of a clinical protocol. To test this hypothesis, we used the magnetic separation approach to metabolically fractionate the breast cancer cell population into "low-energy" and "high-energy" cell subpopulations. For this purpose, we used auto-fluorescence as an endogenous marker of their energetic state riboflavin (vitamin B2). Using vitamin B2 as a fluorescent indicator, we found that BRCA+ CSCs showed a significant reduction in the population of breast cancer cells to "high-energy", and BRCA- CSCs reduced the number of breast cancer cells in the population to "low-energy" (Figure 1).

Thus, BRCA + CSCs reduced the level of "high-energy" population of breast cancer cells to 25\% ( $\mathrm{n}=20$; $\mathrm{p}=0.001)$; the population of "low-energy" BRCA- CSCs was $56 \%(n=20 ; p=0.001)$.

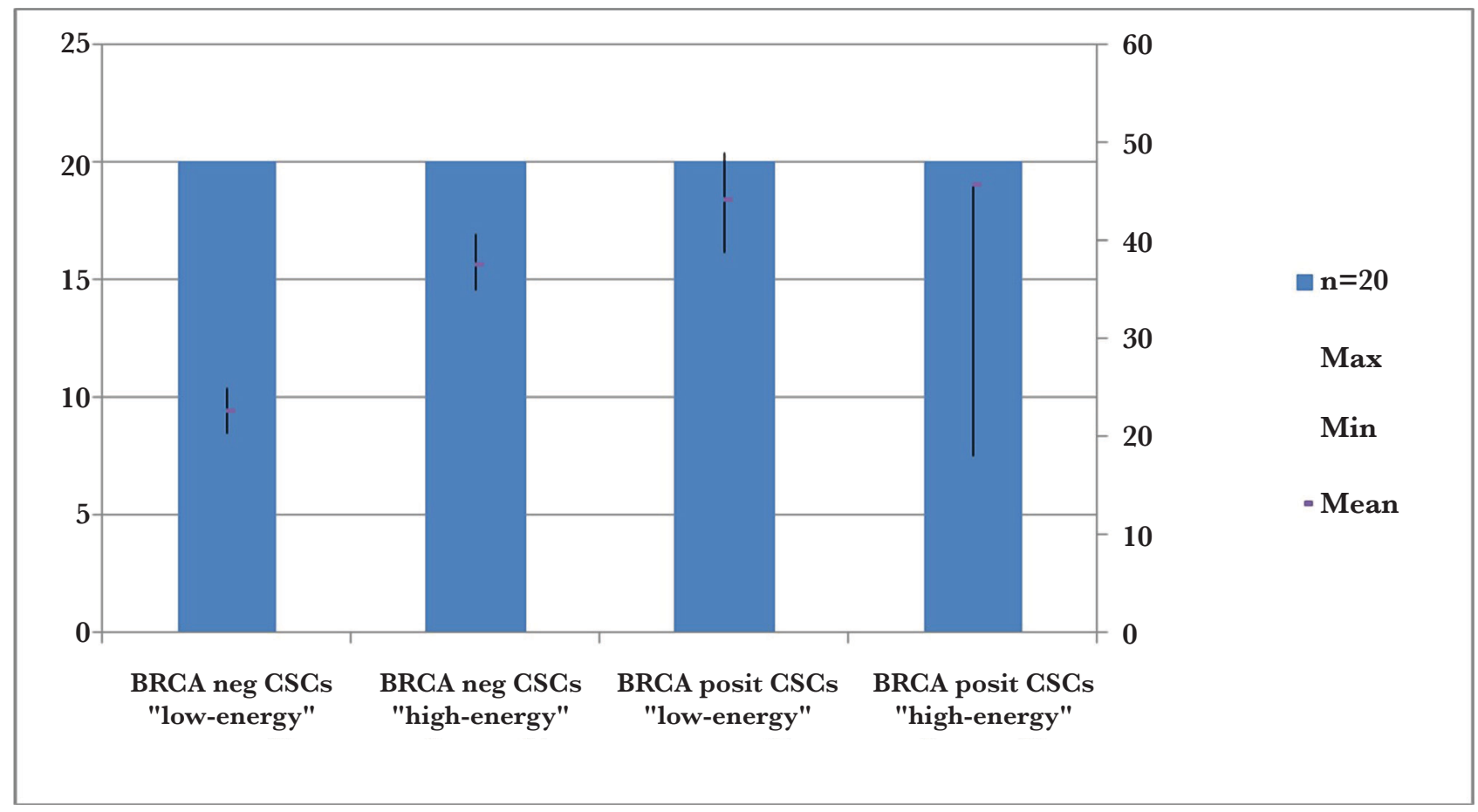




\section{JOURNAL of MEDICINE and LIFE}

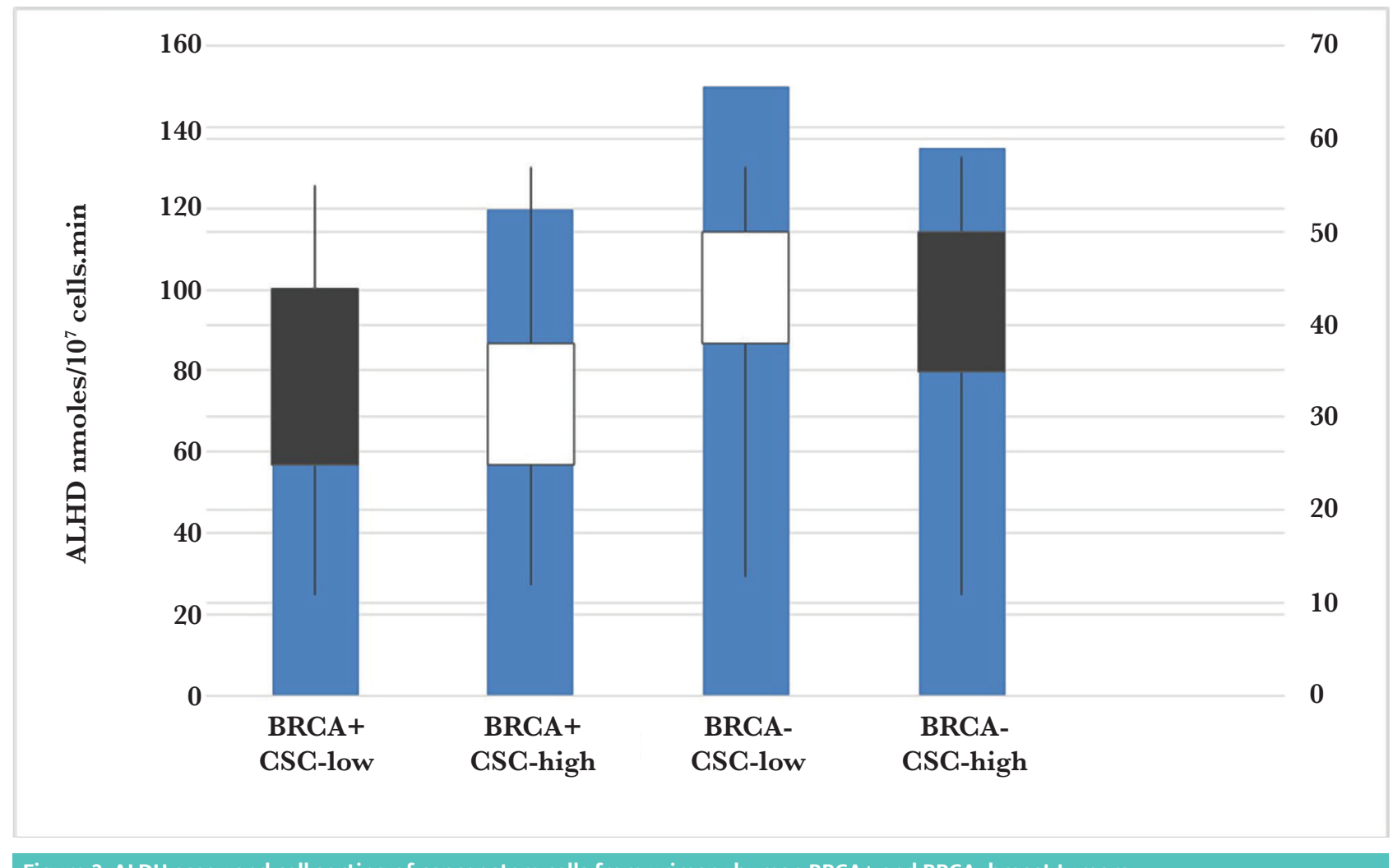

ALDH assay and cell sorting were performed in cancer stem cells from primary human BRCA+ and BRCA- breast tumors. The results of the spectrophotometer cuvettes were noted after the addition of cell lysate, $5 \mathrm{mM} \mathrm{NAD}+$ and $5 \mathrm{mM}$ propionaldehyde as a substrate. The rate of change in absorbance at $340 \mathrm{~nm}$ was measured in 3 replicates over 5 min analyses and showed that the proportion of BRCA+ CSC cells was 35.12 \pm 0.63 nmoles/ 107 cells per min (Figure 2), which was in accordance with the relatively low percentages of CSCs in BRCA+ tumors. ALHD was significantly higher in the CSCs-high BRCA+ breast cancer (45.1 $\pm 2.3 \%)$ and CSCs-low BRCAbreast cancer $(33.6 \pm 1.4 \%)$ cells, compared with the CSCs-low BRCA+ breast cancer $(1.7 \pm 0.53 \%)$ cells.

Loss of BRCA functions results in the genomic instability that eventually results in the oncogenic transformation of non-tumorigenic cells into tumor-initiating cells or cancer stem cells and further tumor evolution. A CSC population possesses a long-term self-renewal capacity, as well as a potential to differentiate into other tumor cell types and initiate tumor growth. As essential regulators of genome stability and gene expression, BRCAl/2 plays an important role in CSCs development and evolution and can be employed in different CSC signaling mechanisms [25-27].

ALDH can be present in the cytosol, the endoplasmic reticulum, mitochondria and even the nucleus, sometimes in more than one place. ALDH is also located in stem cells. During the first stage of life and growth, stem cells have a strong potential to repair the internal system.

They can develop into different cell types in many tissues and divide to restore others without limit [3].

High ALDH activity has been noted in liver cells or the bronchial epithelium of smokers caused by carcinogenic aldehydes in cigarette smoke. Ginestier et al. stated that ALDH1 is a marker for stem/progenitor cells of the normal breast. It is also active in stem cells of several tumors like breast cancer or lung cancer. Its activity might be crucial for both stem cell longevity and the resistance of CSCs to chemotherapy [28-31].

ALDH expression has demonstrated itself to be a possibly relevant prognostic marker. For this reason, the subpopulation of cancer $\mathrm{SC}$ s can be a therapeutic target in cases with a poor prognosis, treatment-resistant and recurrent breast cancer. At the same time, the $\mathrm{ALDH}+$ population isolated from breast tumor specimens displayed a high tumorigenic potential in mice models [32-35]. BRCA1 plays a role in the self-renewal of progenitor cells, a fact which was demonstrated by serial passaging of BRCA1- deficient normal mammary epithelial cells where the number of mammospheres increased with the number of passages [36-38]. 


\section{CONCLUSION}

Accumulating evidence suggests that CSCs exist as a subpopulation of quiescent cells within the dominant tumor bulk of heterogeneous tumor cells. The research on self-renewal pathways that regulate the self-renewal of breast stem cells has led to a clearer picture of how dysregulation of these pathways may lead to carcinogenesis. Furthermore, these pathways may provide targets for breast cancer prevention and therapy. Indeed, recent evidence suggests that key oncogenic pathways known to be dysregulated in breast cancer also regulate stem-cell behavior. Breast cancer from BRCA mutation carriers harbor more "high-energy" cell subpopulations than "low-energy", and have a more aggressive phenotype.

\section{ACKNOWLEDGMENTS}

\section{Ethical approval}

The approval for this study was obtained from the Ethics Committee of the Ukraine Association of Biobank licensed by the Ministry of Health of Ukraine (no. 1813, 04/10/2018) and from the National Authority responsible for biobank cord blood, cell and tissue therapy (no. 1231 27/03/2019).

\section{Consent to participate}

Written informed consent was obtained from the participants.

\section{Conflict of interest}

The authors declare that there is no conflict of interest.

\section{REFERENCES}

1. Banys-Paluchowski M, Reinhardt F, Fehm T. Disseminated Tumor Cells and Dormancy in Breast Cancer Progression. Adv Exp Med Biol. 2020; 1220:35-43. doi: 10.1007/978-3-030-35805-1_3.

2. El Abbass K.A., Abdellateif M.S., Gawish A.M., Zekri A.N., Malash I., Bahnassy A.A. The Role of Breast Cancer Stem Cells and Some Related Molecular Biomarkers in Metastatic and Nonmetastatic Breast Cancer. Clin Breast Cancer. 2020 Mar 12. pii: S1526-8209(19)30756-6. doi: 10.1016/j.clbc.2019.11.008.

3. Gligorijevic, B., Kedrin, D., Segall, J. E., Condeelis, J., and van Rheenen, J. (2009). Dendra2 photoswitching through the mammary imaging window. J. Vis. Exp. 2009:1278. doi: $10.3791 / 1278$

4. Hughes, K., and Watson, C. J. (2018). Sinus-like dilatations of the mammary milk ducts, Ki67 expression, and CD3-positive T lymphocyte infiltration, in the mammary gland of wild European rabbits during pregnancy and lactation. J. Anat. 233, 266-273. doi: 10.1111/joa.12824.

5. Wang Z, Jiang Q, Dong C. Metabolic reprogramming in triple-negative breast cancer. Cancer Biol Med. 2020 Feb 15;17(1):44-59. doi: 10.20892/j.issn.2095-3941.2019.0210.

6. Alieva, M., Ritsma, L., Giedt, R. J., Weissleder, R., and van Rheenen, J. (2014). Imaging windows for long-term intravital imaging: general overview and technical insights. Intravital 3:e29917. doi: 10.4161/intv.29917

7. Andresen, V., Alexander, S., Heupel, W. M., Hirschberg, M., Hoffman, R. M., and Friedl, P. (2009). Infrared multiphoton microscopy: subcellular-resolved deep tissue imaging. Curr. Opin. Biotechnol. 20, 54-62. imaging. Gurr. Opin. Biotechnol. 208

8. Chakrabarti, R., Celià-Terrassa, T., Kumar, S., Hang, X., Wei, Y., Choudhury, A., et al. (2018). Notch ligand DIll mediates cross-talk between mammary stem cells and the macrophageal niche. Science 360:aan4153. doi: 10.1126/science.aan4153

9. Chen, L., Li, G., Li, Y., Li, Y., Zhu, H., Tang, L., et al. (2017). UbasM: An effective balanced optical clearing method for intact biomedical imaging. Sci. Rep. 7:12218. doi: 10.1038/s41598-017-12484-3

10. Chen, Y., Shen, Q., White, S. L., Gokmen-Polar, Y., Badve, S., and Goodman, L. J. (2019). Three-dimensional imaging and quantitative analysis in CLARITY processed breast cancer tissues. Sci. Rep. 9:5624. doi: 10.1038/s41598-019-41957-w
11. Coste A., Oktay, M. H., Condeelis, J. S., and Entenberg, D. (2019). Intravital Imaging Techniques for Biomedical and Clinical Research. Cytom. Part A cyto.a.23963. doi: $10.1002 /$ cyto.a. 23963

12. Davis, F. M., Lloyd-Lewis, B., Harris, O. B., Kozar, S., Winton, D. J., Muresan, L., et al. (2016). Single-cell lineage tracing in the mammary gland reveals stochastic clonal dispersion of stem/progenitor cell progeny. Nat. Commun. 7:13053. doi: $10.1038 /$ ncomms 13053

13. Ebrahim, S., and Weigert, R. (2019). Intravital microscopy in mammalian multicellular organisms. Curr. Opin. Cell Biol. 59, 97-103. doi: 10.1016/j.ceb.2019.03.015

14. Egeblad, M., Ewald, A. J., Askautrud, H. A., Truitt, M. L., Welm, B. E., Bainbridge, E., et al. (2008). Visualizing stromal cell dynamics in different tumor microenvironment by spinning disk confocal microscopy. DMM Dis. Model. Mech. 1, 155-167. doi: 10.1242/dmm.000596

15. Elias, S., Morgan, M. A., Bikoff, E. K., and Robertson, E. J. (2017). Long-lived unipotent Blimp1-positive luminal stem cells drive mammary gland organogenesis throughout adult ife. Nat. Commun. 8:1714 doi: 10.1038/s41467-017-01971-w

16. Follain, G., Mercier, L., Osmani, N. L., SeibastienHarlepp, S., and Goetz, J. G. (2017). Seeing i believing-multi-scale spatio-temporal imaging towards in vivo cell biology. J. Cell Sci. 130, 23-38. doi: 10.1242/jcs.189001.

17. Gramatiuk, S.M., Bagmut, I.Y., Sheremet, M.I., Lazaruk, O.V., Moroz, P.V. Mitochondrial dysfunction of mesenchymal stem cells isolated from blood with type 2 diabetic patients. Romanian Journal of Diabetes, Nutrition and Metabolic Diseases, 2020, 27(4), pp. 309-315

18. Gligorijevic, B., Bergman, A., and Condeelis, J. (2014) Multiparametric classification links tumor microenvironments Multiparametric classification links tumor microenvis.
with tumor cell phenotype. PLoS Biol. 12:e1001995. with tumor cell phenotype. PLOS Bio.
doi: $10.1371 /$ journal.pbio.1001995.

19. Hama, H., Hioki, H., Namiki, K., Hoshida, T., Kurokawa, H., Ishidate, F., et al. (2015). ScaleS: an optical clearing palette for biological imaging. Nat. Neurosci. 18, 1518-1529. doi: 10.1038/nn.4107

20. Nadine Nelson, Karoly Szekeres, Denise Cooper, Tomar Ghansah. Preparation of Myeloid Derived Suppressor Cells (MDSC) from Naive and Pancreatic Tumor-bearing Mice using Flow Cytometry and Automated Magnetic Activated Cell Sorting (AutoMACS). J Vis Exp. 2012; (64): 3875. Published online 2012 Jun 18. doi: 10.3791/3875.

21. Yu-Chen Hu, Jen-Te Lu, Yao-Chi Chung. Highdensity cultivation of insect cells and production of recombinant baculovirus using a novel oscillating bioreactor.
J. Cytotechnology. 2003 Sep; 42(3): 145-153. doi 10.1023/B:CYTO. 0000015841.98225.27

22. Hitchcock, J. R., Hughes, K., Harris, O. B., and Watson, C. J. (2019). Dynamic architectural interplay between leucocytes and mammary epithelial cells. FEBS J. 287:febs. 15126 . doi: $10.1111 /$ febs. 15126

23. Hou B., Zhang, D., Zhao, S., Wei, M., Yang, Z., Wang, S. et al. (2015). Scalable and DiI-compatible optical clearance of the mammalian brain. Front. Neuroanat. 9:19 doi: 10.3389/fnana.2015.00019

24. Inman, J. L., Robertson, C., Mott, J. D., and Bissell, M. J. (2015). Mammary gland development: cell fate specification, stem cells and the microenvironment. Dev. 142, 1028-1042. doi: $10.1242 /$ dev. 087643

25. Jardé, T., Lloyd-Lewis, B., Thomas, M., Kendrick, H., Melchor, L., Bougaret, L., et al. (2016). Wnt and Neuregulin 1/ErbBsignalling extends 3D culture of hormone responsive mammary organoids. Nat. Commun. 7:13207. responsive mammary organ
doi: $10.1038 /$ ncomms 13207

26. Katie McDole, A., Guignard, L., Amat, F., Turaga, S C., Branson, K., and Keller Correspondence, P. J. (2018). In Toto imaging and reconstruction of post-implantation mouse development at the single-cell level. Cell 175, 859.e33-876. e33. doi: 10.1016/j.cell.2018.09.031

27. Ke M. T., Nakai, Y., Fujimoto, S., Takayama, R., Yoshida, S., Kitajima, T. S., et al. (2016). Super-resolution mapping of neuronal circuitry with an index-optimized clearing agent. Cell Rep. 14, 2718-2732. doi: 10.1016/j.celrep.2016.02.057.

28. Ginestier C, Hur MH, Charafe-Jauffret E, Monville F, Dutcher J, Brown M, Jacquemier J, Viens P, Kleer CG, Liu S, Schott A, Hayes D, Birnbaum D, Wicha MS, Dontu G. ALDH1 is a marker of normal and malignant human mammary stem cells and a predictor of poor clinical mammary stem cells and a predictor of poor clinical
outcome. Cell Stem Cell. 2007 Nov; 1(5):555-67. outcome. Cell Stem Cell. 2007 Nov; 1(5):555-67. doi: 10.1016/j.stem.2007.08.014.

29. Arfaoui A, Rioualen C, Azzoni V, Pinna G, Finetti P, Wicinski J, Josselin E, Macario M, Castellano R, Léonard-Stumpf C, Bal A, Gros A, Lossy S, Kharrat M, Collette Y, Bertucci F, Birnbaum D, Douik H, Bidaut G, Charafe-Jauffret E, Ginestier. A genome-wide RNAi screen reveals essential therapeutic targets of breast cancer stem cells. EMBO Mol Med. 2019 Oct;11(10):e9930. doi: 10.15252/emmm.201809930. Epub 2019 Sep 2.

30. Lilja A. M., Rodilla V., Huyghe, M., Hannezo, E., Landragin, C., Renaud, O., et al. (2018). Clonal analysis of Landragin, C., Renaud, O., et al. (2018). Clonal analysis of stem cells that retain long-term plasticity in the embryonic 


\section{JOURNAL of MEDICINE and LIFE}

mammary gland. Nat. Cell Biol. 20, 677-687. doi: 10.1038/s41556-018-0108-1

31. Lloyd-Lewis, B., Davis, F. M., Harris, O. B., Hitchcock, J. R., Lourenco, F. C., Pasche, M., et al. (2016). Imaging the mammary gland and mammary tumours in 3D: optical tissue clearing and immunofluorescence methods. Breast Cancer Res. 18:127. doi: 10.1186/s13058-016-0754-9

32. Lloyd-Lewis, B., Davis, F. M., Harris, O. B., Hitchcock, J. R., and Watson, C. J. (2018). Neutral lineage tracing of proliferative embryonic and adult mammary stem/progenitor cells. Development 145:dev164079. doi: 10.1242/dev. 164079

33. Lloyd-Lewis, B., Mourikis, P., and Fre, S. (2019). Notch signalling: sensor and instructor of the microenvironment to coordinate cell fate and organ morphogenesis. Curr. Opin Cell Biol. 61, 16-23. doi: 10.1016/J.CEB.2019.06.003
34. Masedunskas, A., Chena, Y., Stussman, R., Weigert, R., and Mather, I. H. (2017). Kinetics of milk lipid drople transport, growth, and secretion revealed by intravital imaging: Lipid droplet release is intermittently stimulated by oxytocin. Mol. Biol. Cell 28, 935-946.

doi: 10.1091/mbc.E16-11-0776

35. Matryba, P., Kaczmarek, L., and Gołąb, J. (2019). Advances in ex situ tissue optical clearing. Laser Photon. Rev. 13:1800292. doi: 10.1002/lpor.201800292

36. Nobis, M., Warren, S. C., Lucas, M. C., Murphy, K. J, Herrmann, D., and Timpson, P. (2018). Molecular mobility Herrmann, D., and Timpson, P. (2018). Molecular mobility
and activity in an intravital imaging setting - Implications for cancer progression and targeting. J. Cell Sci. 131:jcs.206995. doi: $10.1242 /$ jcs. 206995
37. Perrin, L., Bayarmagnai, B., and Gligorijevic, B. (2019). Frontiers in intravital multiphoton microscopy of cancer Cancer Rep. 3, 1-22. doi: 10.1002/cnr2.1192

38. Christophe Ginestier, Min HeeHur, Emmanuelle Charafe-Jauffret, Florence Monville, Julie Dutcher, Marty Brown. ALDH1 Is a Marker of Normal and Malignant Human Mammary Stem Cells and a Predictor of Poor Clinical Outcome. Cell Stem Cell. 2007 Nov;1(5):555-67. doi: $10.1016 /$ j.stem.2007.08.014. 